$$
\text { استخدام مثسعة المفاتيح الساكنة في بلاء المحرك الحثي أحادي الطور }
$$

الملخص

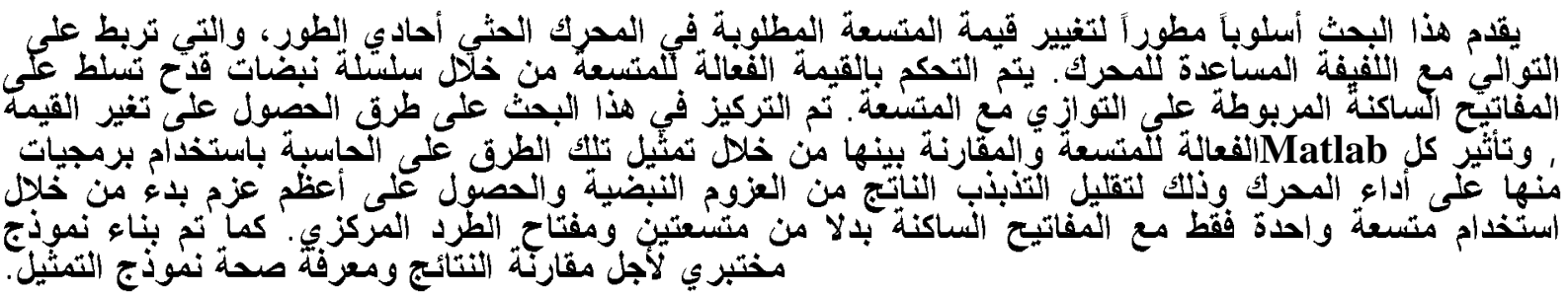

\title{
Starting of the Single Phase Induction Motor using Static Switched Capacitor
}

\author{
Yasser A. Mahmood \\ Assist. Lecturer / Elect. Eng. / University of Mosul
}

\begin{abstract}
This paper provides a method of changing the effective value of the capacitor for starting a single phase induction motor starting. This capacitor is connected in series with the auxiliary winding. Its effective value can be controlled by static switches in parallel with the capacitor through a series of pulses. The focus was on finding ways of obtaining the best effective capacitor during starting condition through computer simulation software using (Matlab). By using this method, only one capacitor is used for both the starting and running conditions and a similar starting performance can be obtained when compared with the conventional method using two capacitors. The computer simulation results are validated by building a laboratory model and comparison of results.
\end{abstract}

Key words: single phase induction motor, switched capacitor, variable capacitor, starting conditions. 


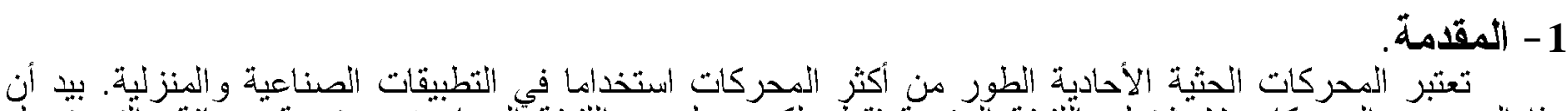

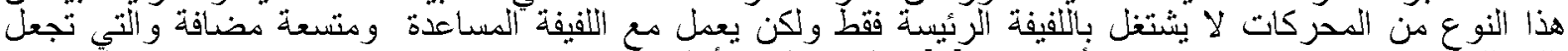

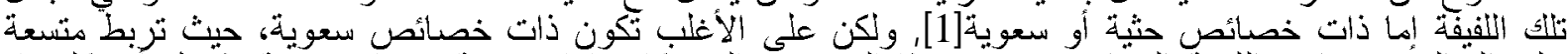

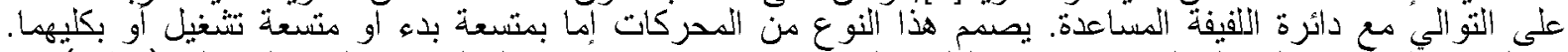

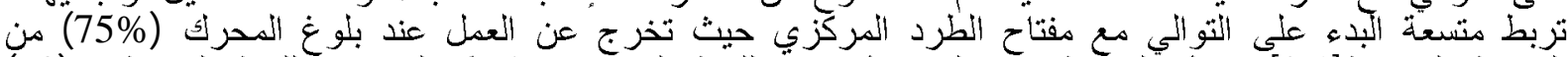

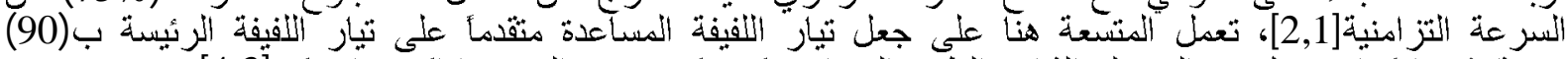

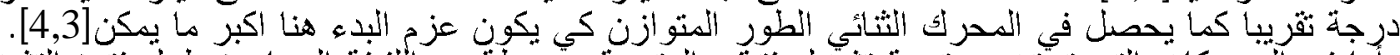

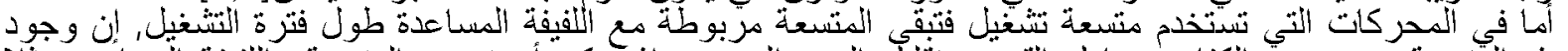

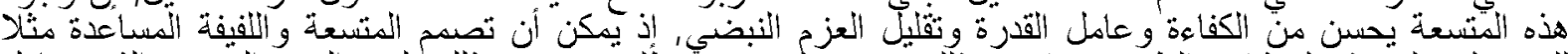

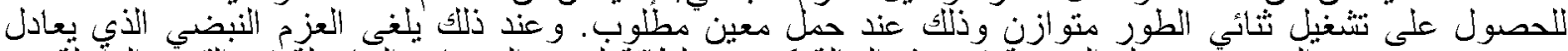

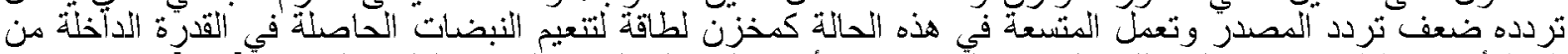

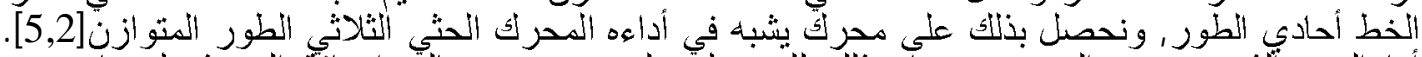

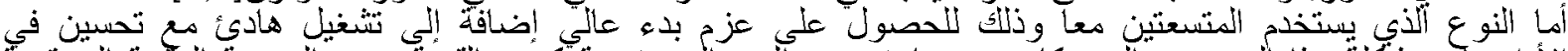

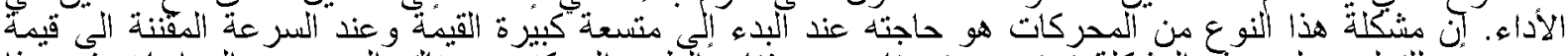

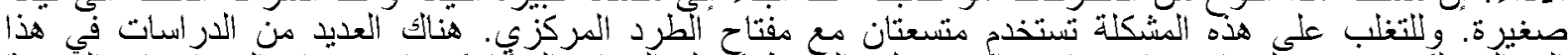

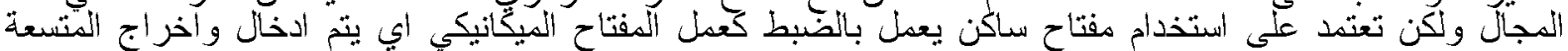

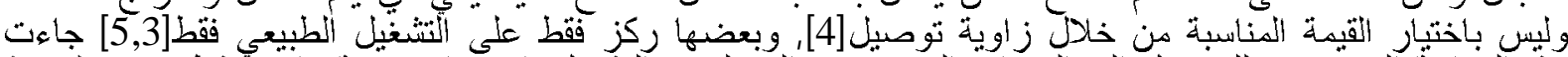

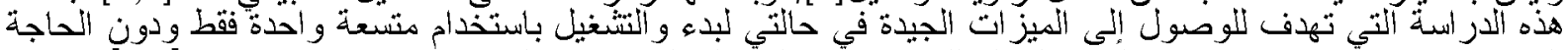

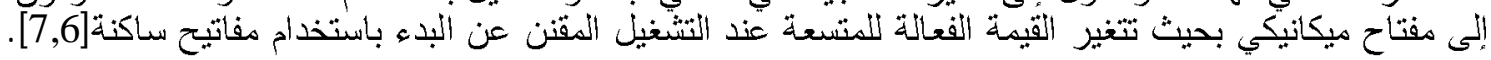

2 - 2 القيمة الفعائة للمتسعة.

أستتد هذا البحث على بذاء نموذج حاسوبي باستخدام برمجيات (Matlab \Power System Blockset v7.6) وهي احد أشهر الرزم البرمجية (software packages) التي تستخدم لتمثيل وتحليل المنظومات الكهرباتئية. تم بناء

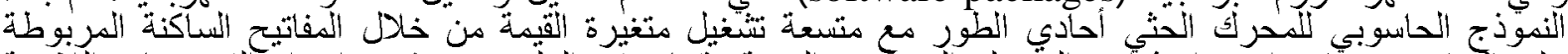

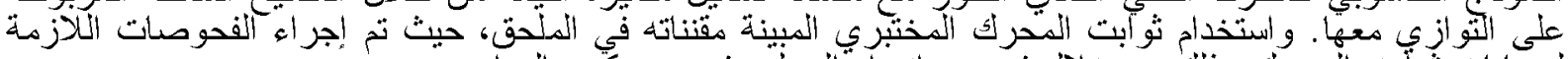

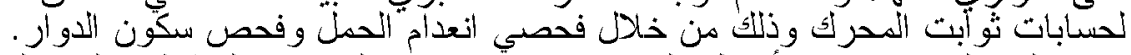

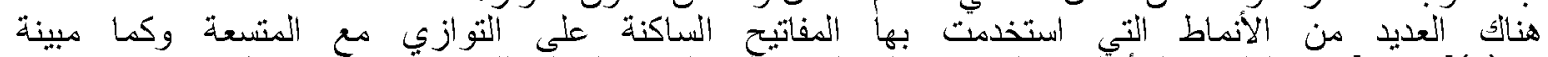

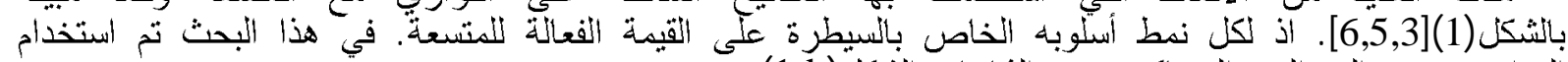

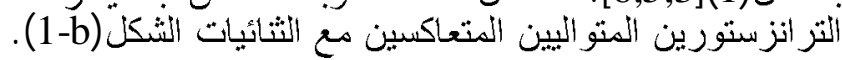

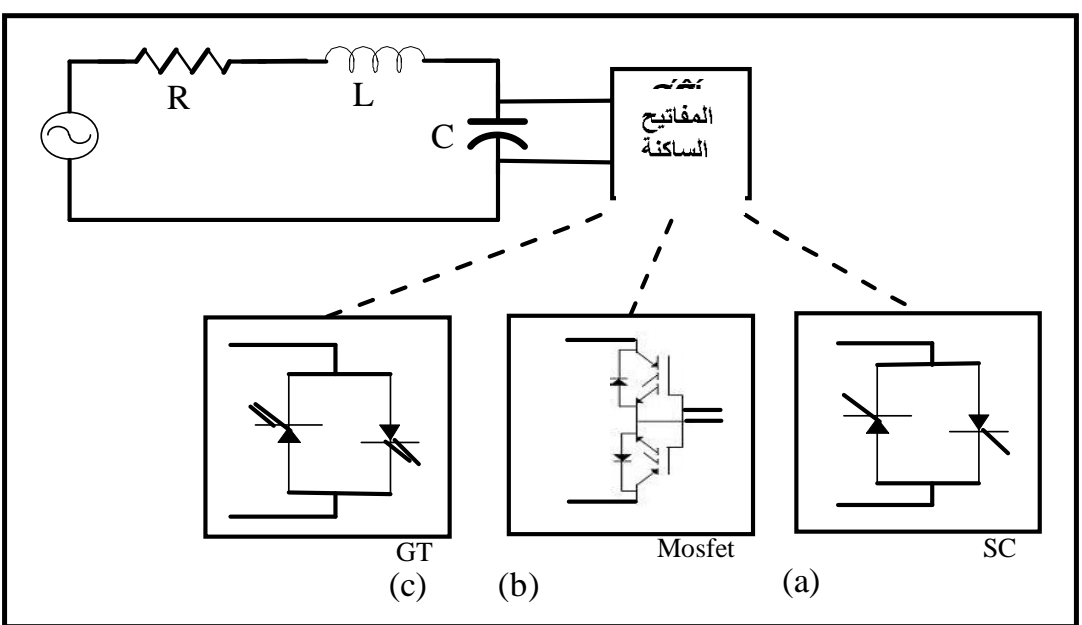

الثكل(1): دائرة RLC مع المفاتيح الساكنة لتغيير النعة.

أما استخدام الثايروستورين المتعاكسين (a) Back-to-Back thyristor) في تغيير قيمة السعة فيسبب مشكلة

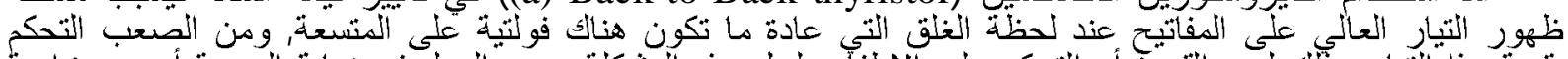

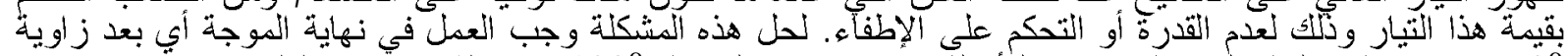

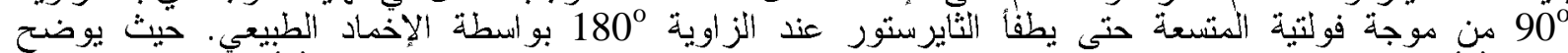

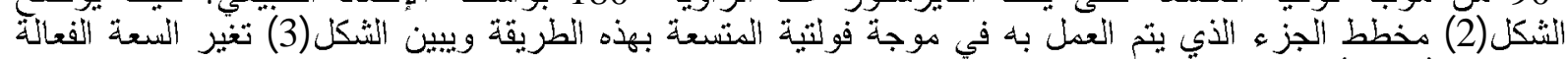
في دائرة (RLC)

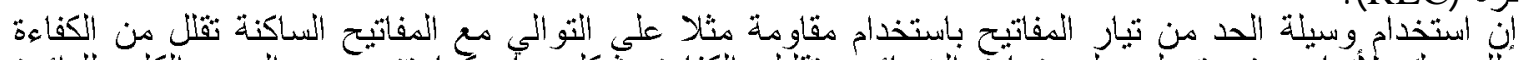

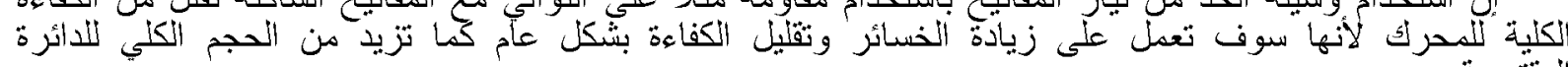
المقترحة. 


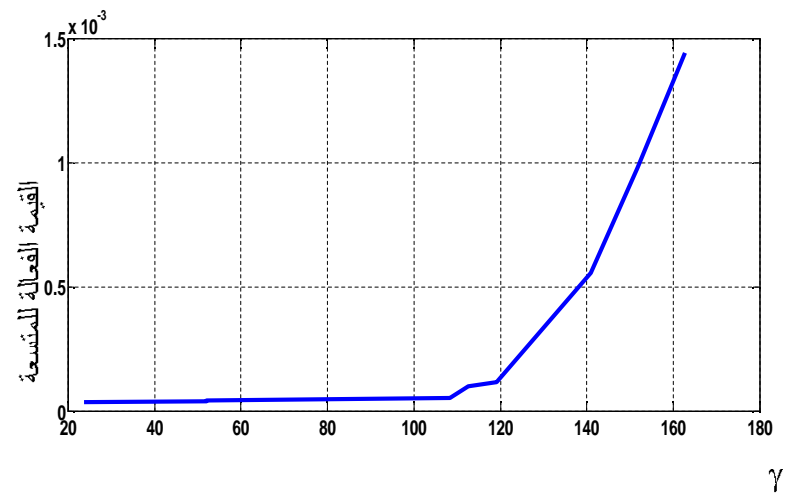

الثنكل(3): علاقة المتسعة الفعالة هع تغير زاوية التوصيل باستخدام الثايرستورين المتعاكسين.

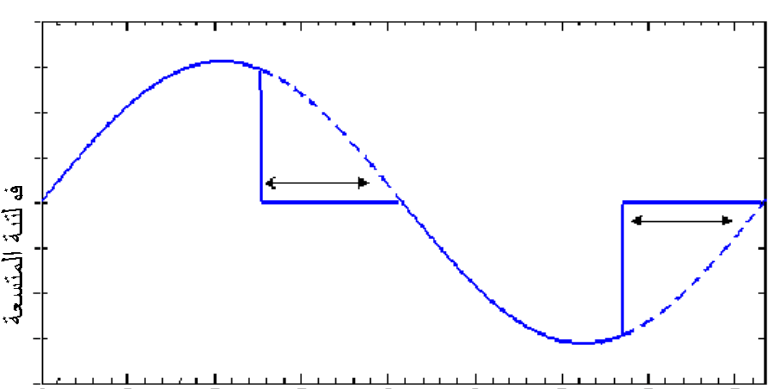

ز يعمل بد الثاير ستو ( ان المتعاكسان.

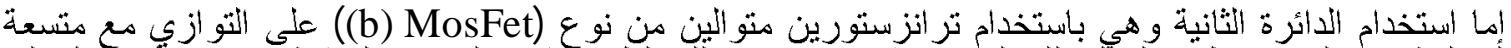

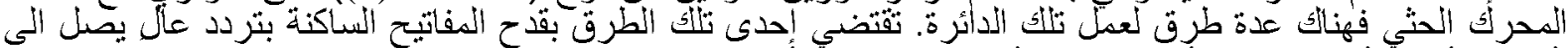

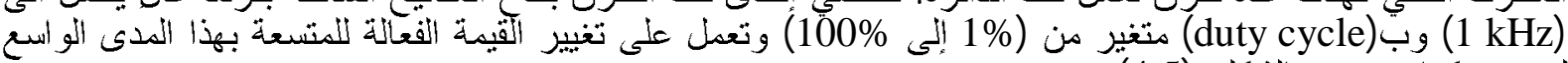
لتنغيير وكما هوضح بالثكلين(4,5).
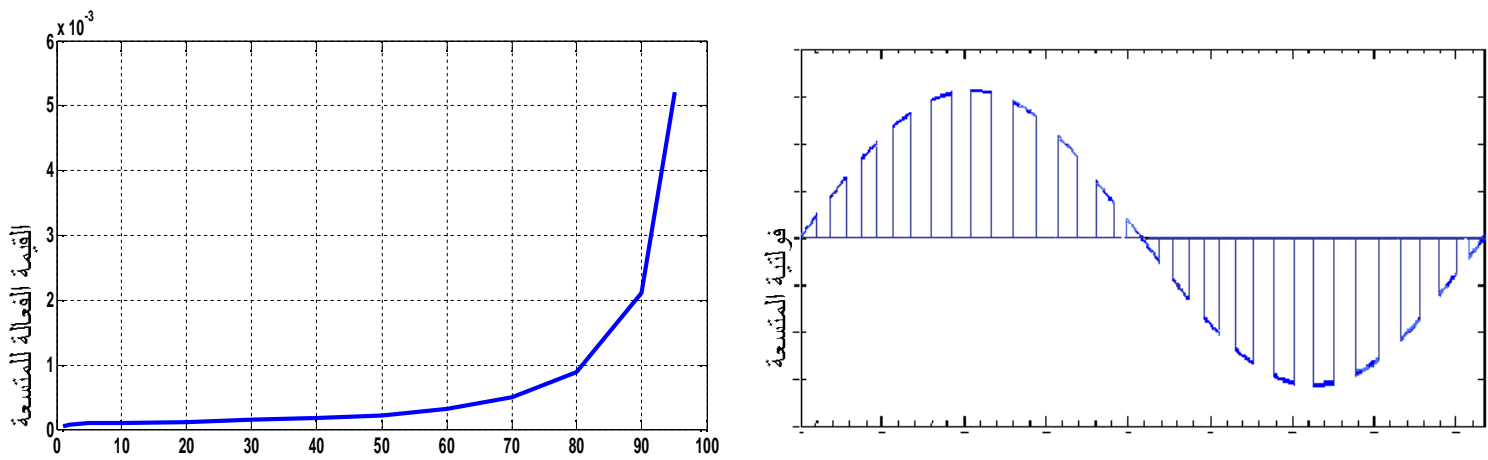

Duty
cycle

الثنكل(5): علاقة تغير قيمة المتسعة الفعالة مع تغير النسبة الكئوية للغلق على الزهن الكلي (duty cycle) عند قيمة فئمة

$$
\text { الترانزوستورين المتو اليين بتردد علئوب عملي. }
$$

ويتم التحكم بمدى بقاء إثارة القدح و التي تمثل زاوية التوصبل هنا و التي هن خلالها يتم الحصول على قيم مختلفة

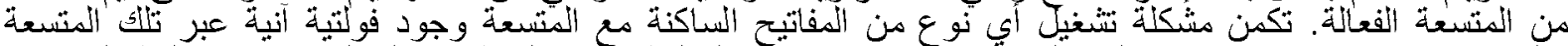

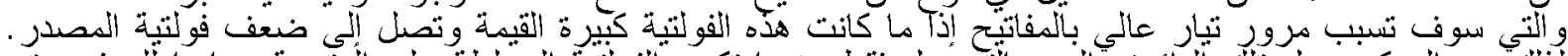

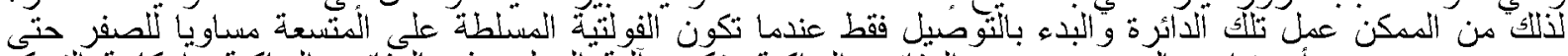

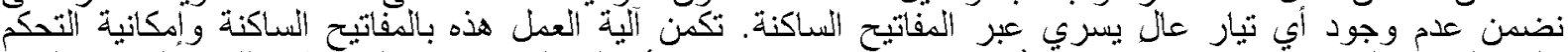

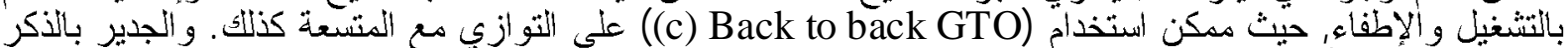

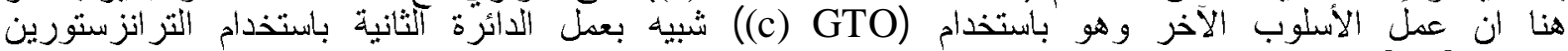
المتو الين[6,5]

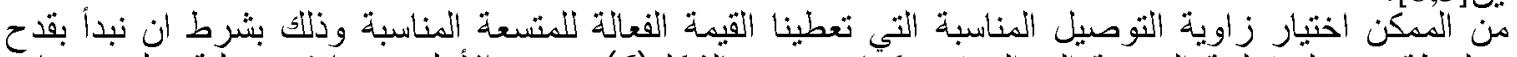

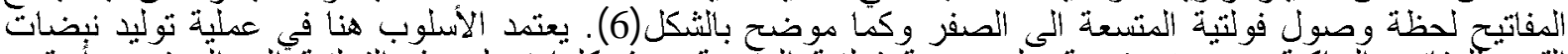

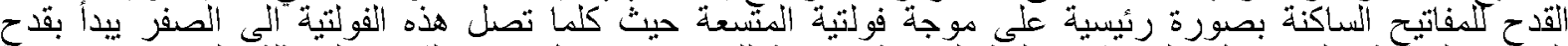

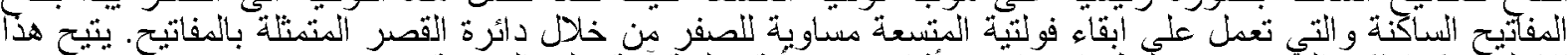

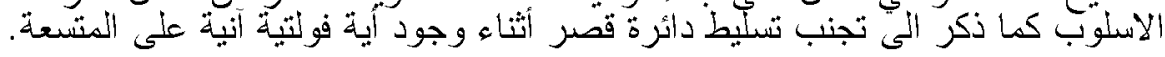

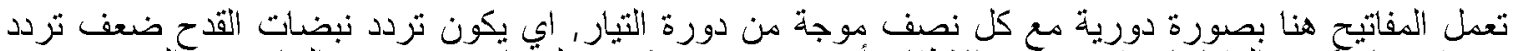

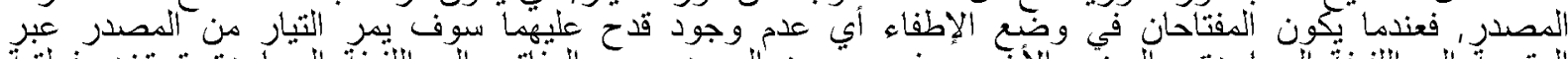

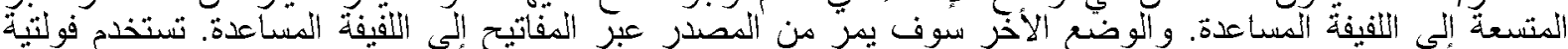

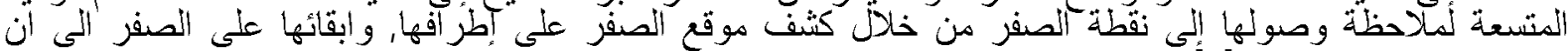

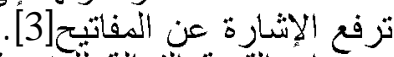

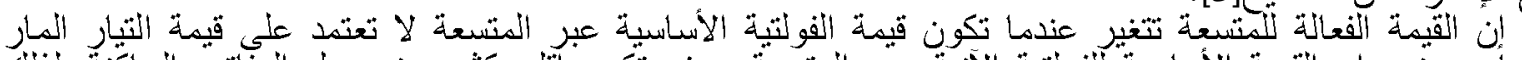

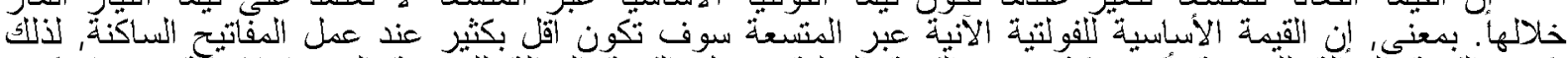

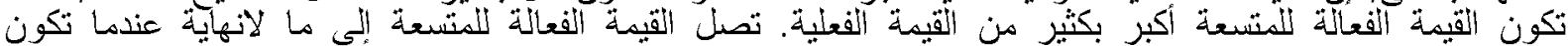

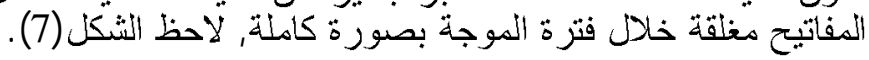




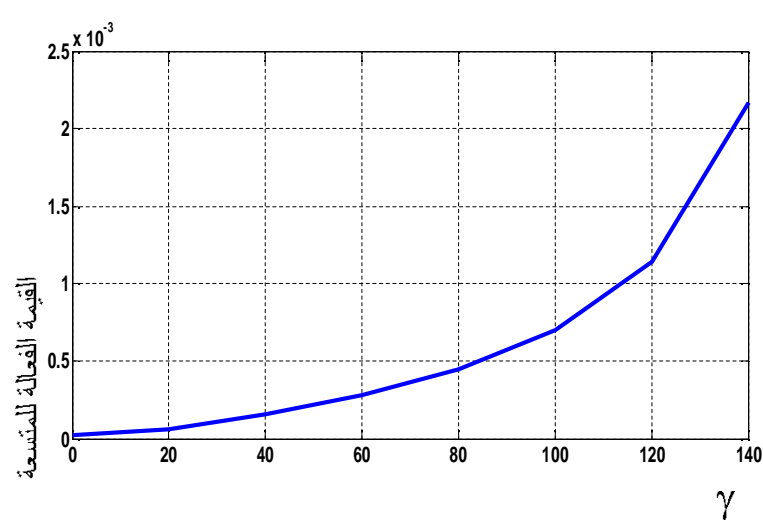

التُكل(7): علاقة تغير القبمة الفعالة للمتسعة مع تغير

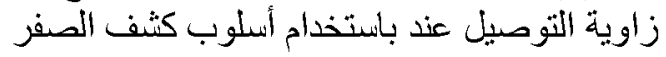

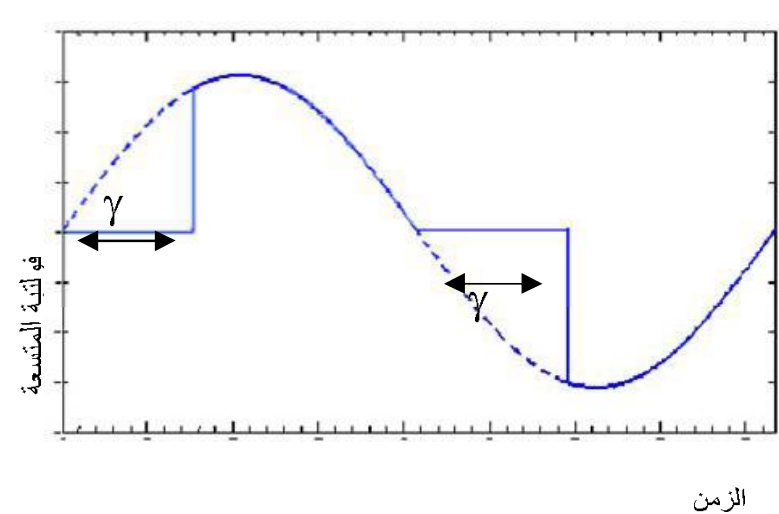

الثكل(6): مخطط يوضح أسلوب عمل مبداً الكثنف عن النّن

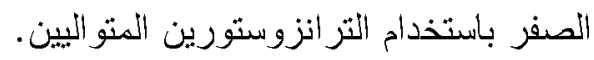

\section{3 - وصف لألية التحكم وتطبيقها.}

يتت تحويل الفوتنية إلبي موجة مربعة حيث يستفاد من الجزأين الموجب والساتب لقدح زوج المفاتيح الساكنة

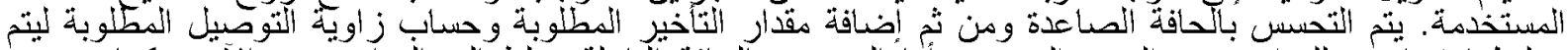

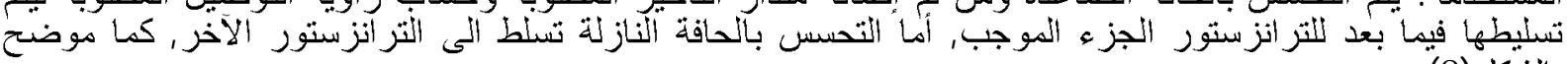

بالثشكل(8).

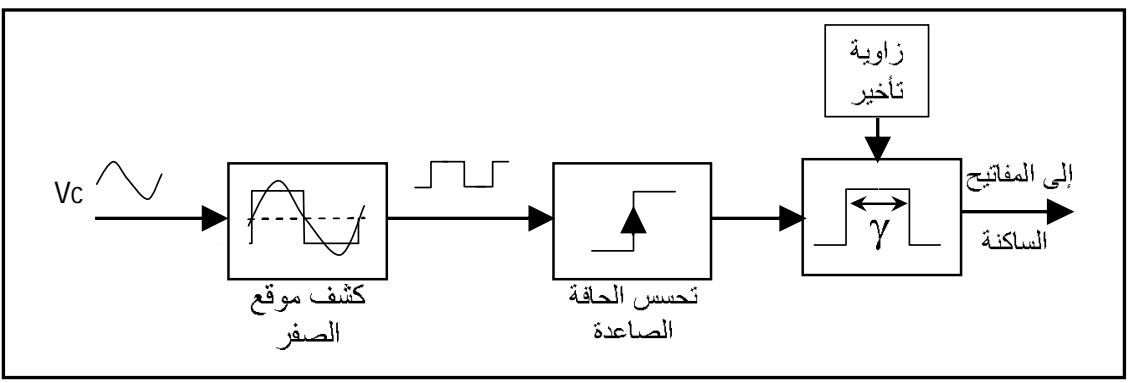

$$
\text { الثكل(8): أسلوب توليد زوايا قدح المفاتيح الساكنة. }
$$

تم تطبيق هذا الأسلوب بتغيير السعة على دائرة (RLC circuit) وتم اخذ نتائج القيمة الفعالة للمتسعة و عامل الفئل

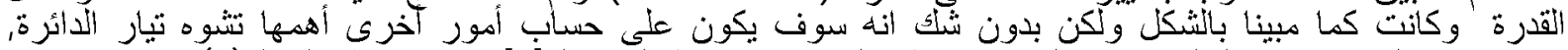

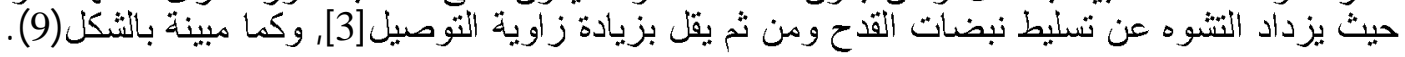

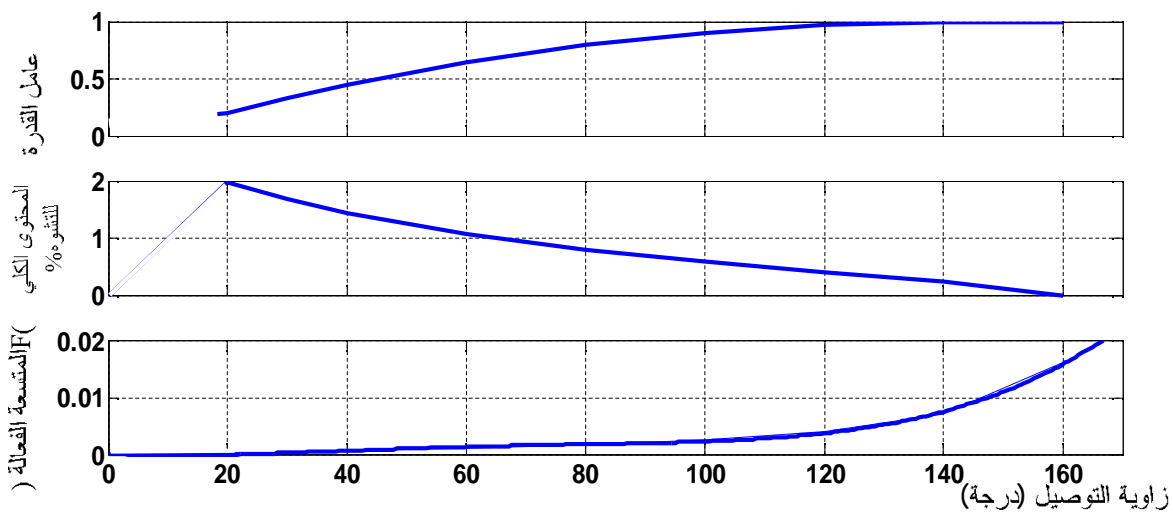

الثكل(9): يوضح تغير قيمة المتسعة الفعالة والمحتوى الكلي للتشوهو وعامل القدرة مع زاوية 
تم تتفيذ هذا الأسلوب لتغنير السعة مع المحرك الحثي أحادي الطور الثكل(10) باستخدام النموذج الحاسوبي وبقيمة

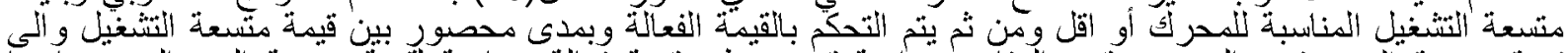

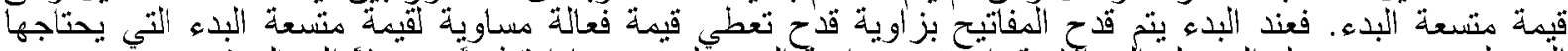

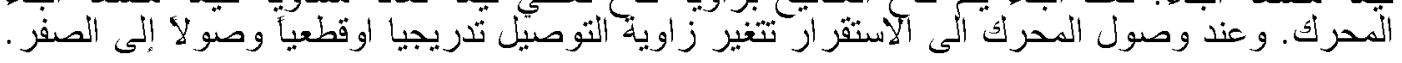

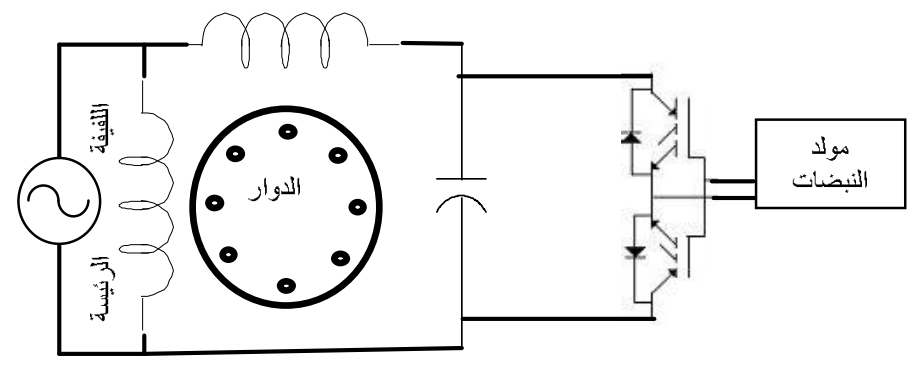

الثنكل (10): دائزة المحر أك الحثي مع المفاتيح الساكنة.

4 - حالة الباءع.

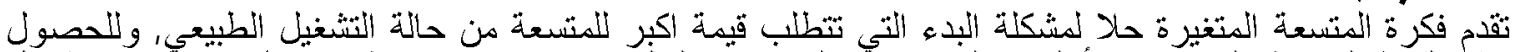

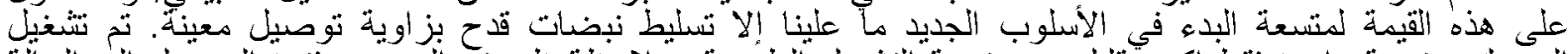

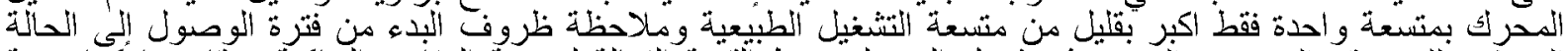

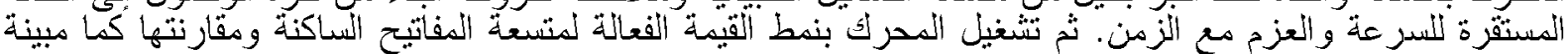

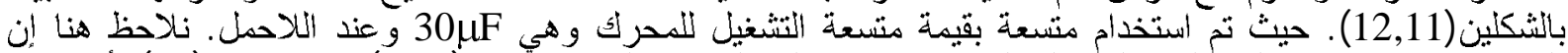

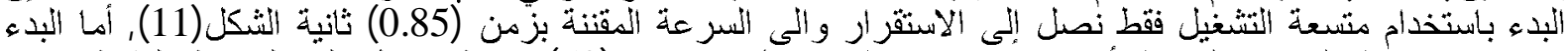

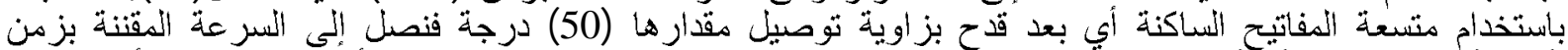

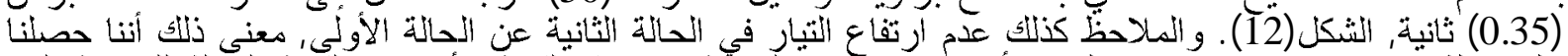

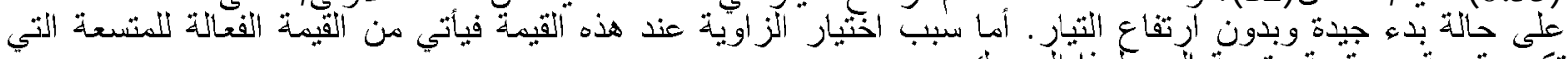
تكون قريية من قيمة متسعة البده كهذا المحرك.

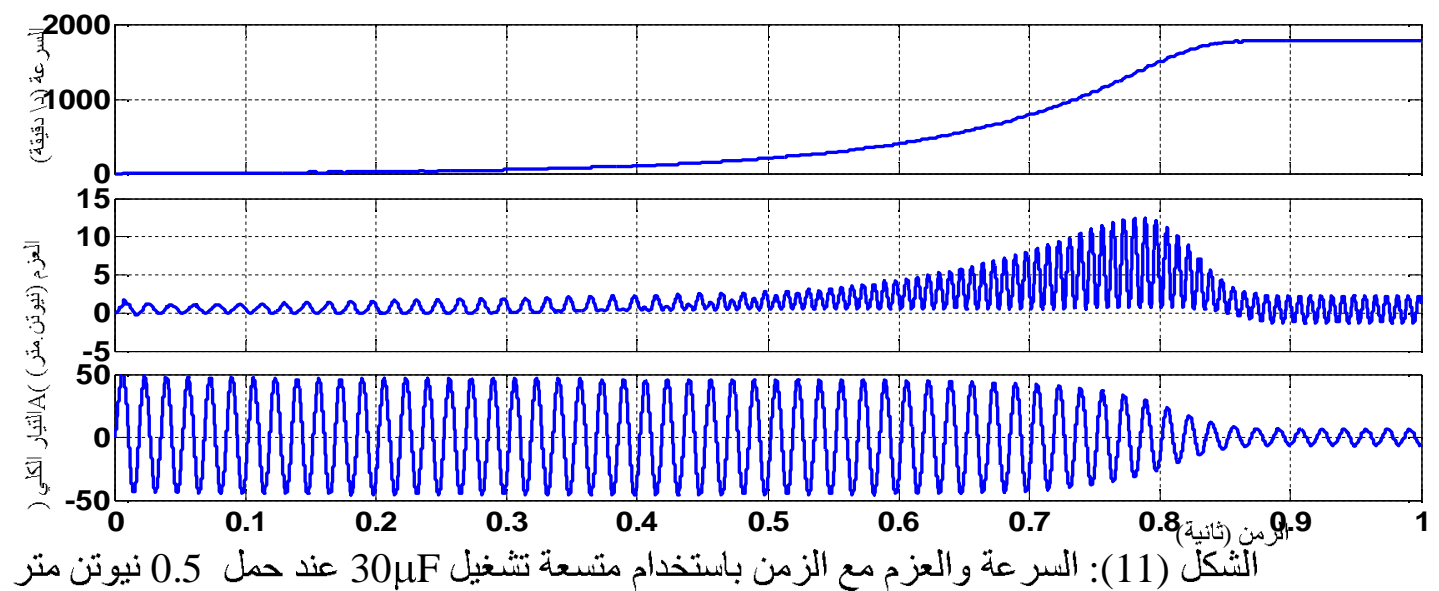

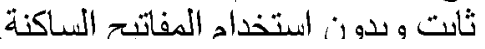

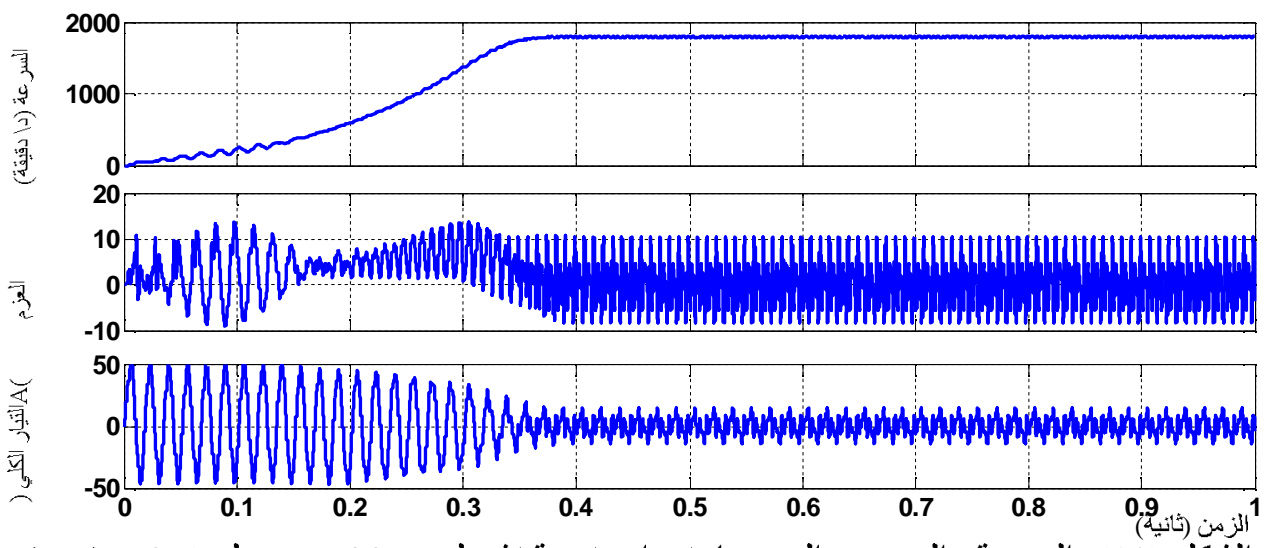

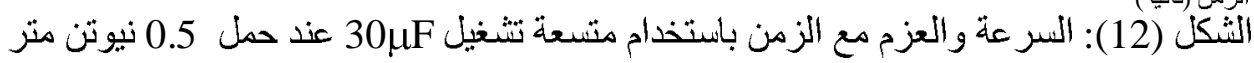
باستخدام المفاتيح كلاكياكنة وبز اوية توصنيل 


\section{5 - فولتية وتيار متسعة المفاتيح الساكنة.}

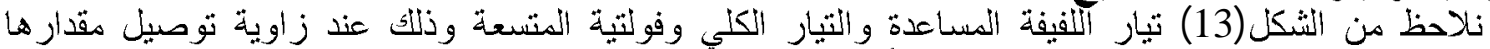

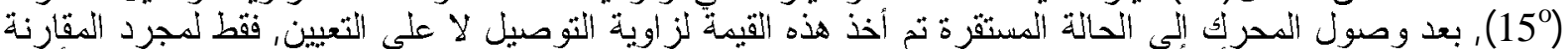

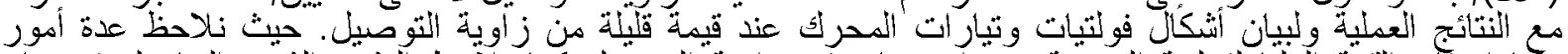

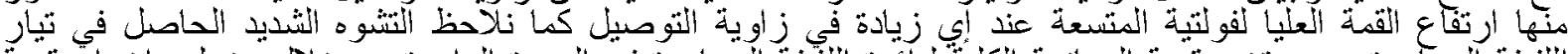

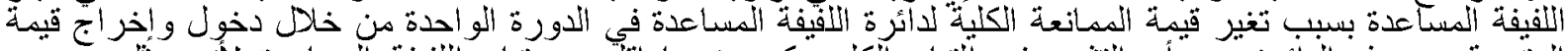

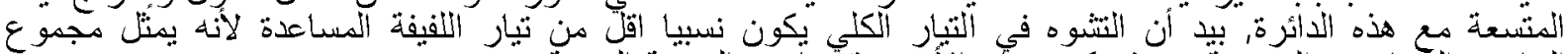

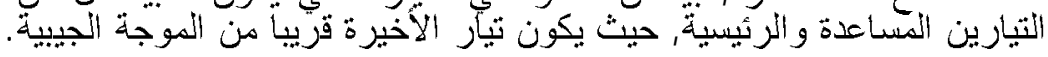
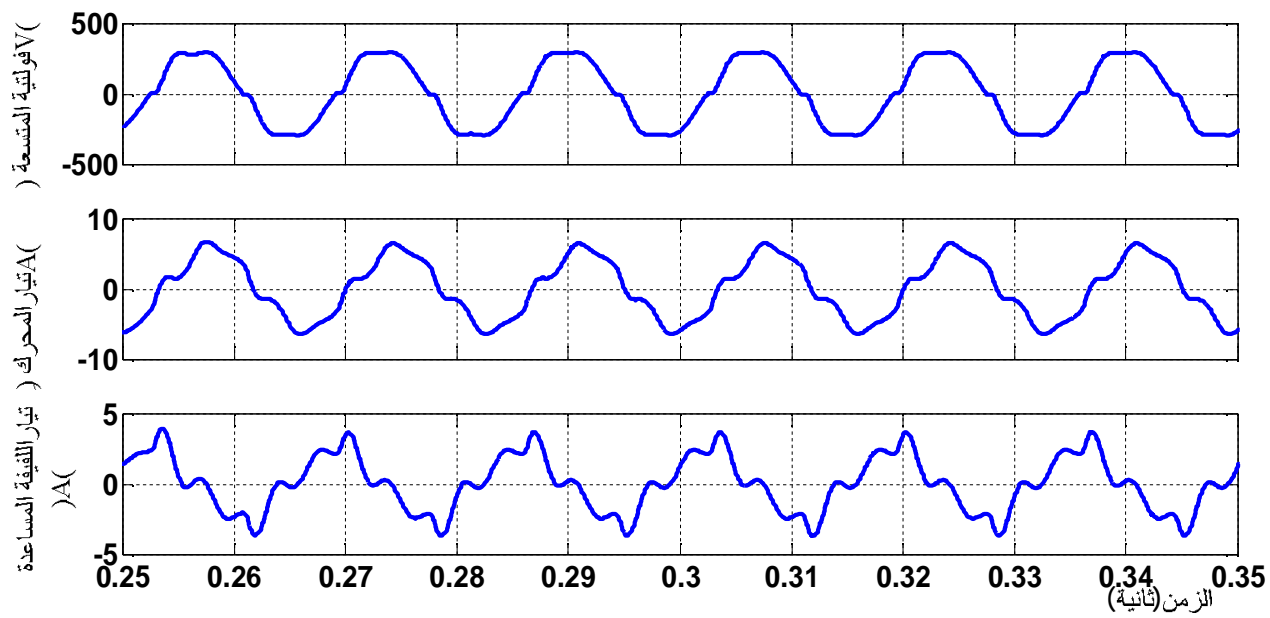

الثكل(13): يوضح موجات فونتية المتسعة والتبار الكلي وتيار اللفيفة المساعدة في الحالة المستقرة

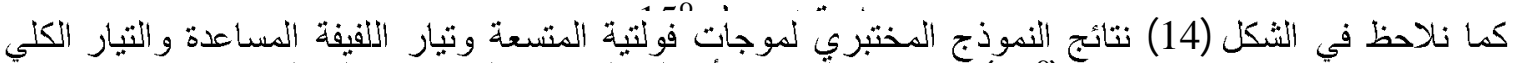

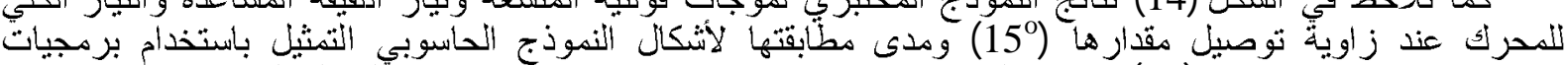

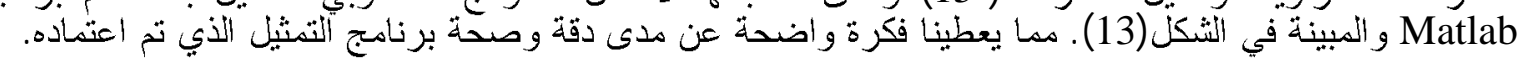
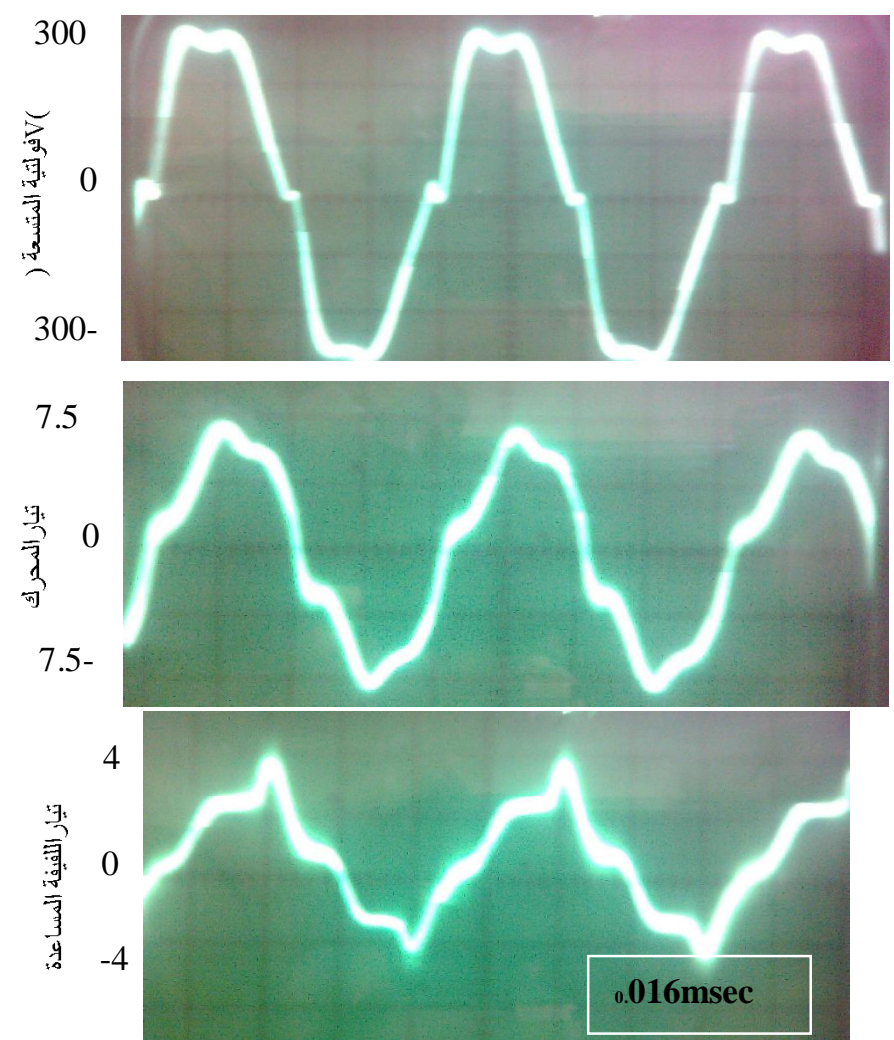

الثكل(14): النتيجة العملية لثكل a) فولتية المتسعة b) تيار المحرك الكلي c) تيار اللفيفة المساعدة وبز اوية توصيل فئنة $15^{\circ}$ 
يقدم هذا البحث نموذجأ لبادئ حركة (Motor Starter) ساكن للمحرك الحتثي أحادي الطور مكوّن من نبائط

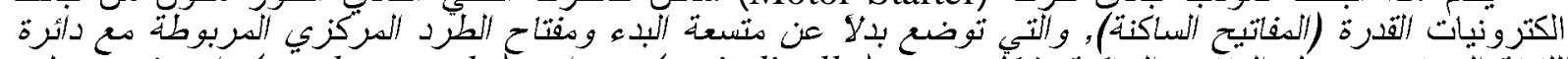

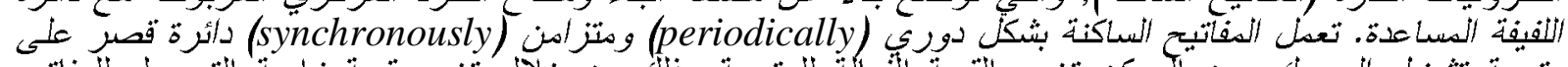

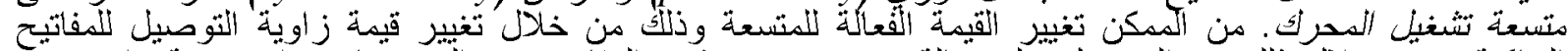

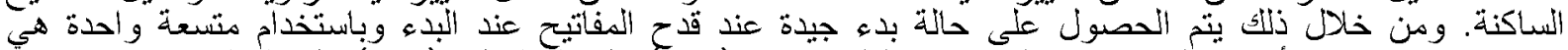

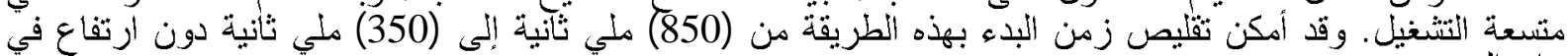
تيار البدء.

$$
7
$$

1.T. Wildi. "Electrical Machines, Drives, and Power system" Pearson Prentice Hall, sixth edition, 2006.

2.S. Ghosh " Electrical Machines " Pearson Education, 2005.

3.S. Sunter, M. Ozdemir, and B. Gumus, " Modeling and Simulation of a Single-Phase Induction Motor with Adjustable Switched Capacitor " $9^{\text {th }}$ International conferences on power electronics and motion control-EPE- PEMC 2000.

4.R. Rabinovici and Z. Keller " New Electronic Starter for Single Phase Induction Motors

" IEEE Transaction on Magnetics, vol 32, No 5, Sept 1996.

5.E. Muljadi, Y. Zhao, T. Liu and T. Lipo " Adjustable ac Capacitor for a Single-Phase

Induction Motor " IEEE Transaction on industry application, vol 29, No 3, May 1993.

6.T. Lettenmaier, D. Novotny and T. Lipo " Single-Phase Induction Motor with an Electronically Controlled Capacitor " IEEE Transaction on industry application, vol 27, No 1, Jan/Feb 1991.

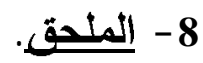

Single phase induction motor:-

RLC circuit:-

$\mathrm{r}_{1 \mathrm{~m}}=0.78 \Omega, \quad \mathrm{L}_{1 \mathrm{~m}}=0.0032 \mathrm{H}$,

$\mathrm{R}=5 \Omega, \mathrm{L}=0.6 \mathrm{H}, \mathrm{C}=$

$20 \mu \mathrm{F}$

$\mathrm{r}_{2}{ }^{\prime}=1.6 \Omega, \quad \mathrm{L}_{2}{ }^{\prime}=0.00318 \mathrm{H}$,

$\mathrm{r}_{1 \mathrm{a}}=3.52 \Omega, \quad \mathrm{L}_{1 \mathrm{a}}=0.0087 \mathrm{H}$,

$\mathrm{a}=1.66$,

$$
\text { تم اجراء البحث في كلية الهنلسة - جامعة الموصل }
$$

